Supporting information for the manuscript

\title{
Adiabatic Electron Affinities of the Polyhydrated Adenine- Thymine Base Pair: A Density Functional Study
}

\author{
Anil Kumar ${ }^{1}$, P.C. Mishra ${ }^{1}$ and Sándor Suhai ${ }^{2}$ \\ ${ }^{1}$ Department of Physics, Banaras Hindu University, Varanasi-221 005, INDIA \\ ${ }^{2}$ Department of Molecular Biophysics, Deutsches Krebsforschungszentrum, Im \\ Neuenheimer Feld 580, 69120 Heidelberg, GERMANY
}

Table 1: Mulliken and Natural Population Analysis (NPA) charges in neutral AT- $5 \mathrm{H}_{2} \mathrm{O}$ calculated using B3LYP/6-31+G** method. ${ }^{\text {a }}$

\begin{tabular}{|c|c|c|c|c|c|c|}
\hline \multirow{2}{*}{ Atom No. } & \multicolumn{3}{|c|}{ Mulliken Charges } & \multicolumn{3}{|c|}{ NPA Charges } \\
\hline & Adenine(A) & Thymine(T) & Water $(\mathrm{W})^{\mathrm{b}}$ & Adenine(A) & Thymine(T) & Water(W) ${ }^{b}$ \\
\hline 1 & -0.4581 & -0.4190 & 0.0007 & -0.6043 & -0.6317 & -0.0108 \\
\hline 2 & 0.3618 & 0.7235 & -0.0321 & 0.2683 & 0.8281 & -0.0078 \\
\hline 3 & -0.4344 & -0.5517 & 0.0352 & -0.5777 & -0.6718 & -0.0209 \\
\hline 4 & -0.3883 & 0.2289 & 0.0057 & 0.3590 & 0.6782 & -0.0155 \\
\hline 5 & 0.4313 & 0.9770 & 0.0090 & 0.0026 & -0.1660 & -0.0074 \\
\hline 6 & 0.2528 & -0.5345 & & 0.4403 & 0.0338 & \\
\hline 7 & -0.5405 & -0.5938 & & -0.5431 & -0.6615 & \\
\hline 8 & 0.3478 & -0.5786 & & 0.2168 & -0.6780 & \\
\hline 9 & -0.3633 & -0.6876 & & -0.5828 & -0.7130 & \\
\hline 10 & -0.6478 & 0.2047 & & -0.8199 & 0.2821 & \\
\hline 11 & 0.3733 & 0.1774 & & 0.4549 & 0.2673 & \\
\hline 12 & 0.3823 & 0.1399 & & 0.4488 & 0.2446 & \\
\hline 13 & 0.1848 & 0.1566 & & 0.2597 & 0.2526 & \\
\hline 14 & 0.3649 & 0.3370 & & 0.4898 & 0.4629 & \\
\hline 15 & 0.1463 & 0.3888 & & 0.2394 & 0.4830 & \\
\hline Total & 0.0129 & -0.0314 & 0.0185 & 0.0518 & 0.0106 & -0.0624 \\
\hline
\end{tabular}

${ }^{\mathrm{a}}$ See Fig. 1a.

${ }^{\mathrm{b}}$ Total charge on the surrounding water molecule. 
Table 2: Mulliken and Natural Population Analysis (NPA) charges in neutral AT- $13 \mathrm{H}_{2} \mathrm{O}$ calculated using B3LYP/6-31+G** method. ${ }^{\mathrm{a}}$

\begin{tabular}{ccccccc}
\hline \multirow{2}{*}{ Atom No. } & \multicolumn{5}{c}{ Mulliken Charges } & \multicolumn{3}{c}{ NPA Charges } \\
\cline { 2 - 6 } & Adenibe(A) & Thymine(T) & Water(W) & Adenine(A) & Thymine(T) & Water(W) $^{\mathrm{b}}$ \\
\hline 1 & -0.3610 & -0.4447 & 0.0024 & -0.6014 & -0.6277 & -0.0042 \\
2 & 0.1696 & 0.7023 & 0.0247 & 0.2676 & 0.8318 & -0.0053 \\
3 & -0.3886 & -0.5242 & -0.0014 & -0.5692 & -0.6612 & -0.0030 \\
4 & -0.1940 & 0.2483 & -0.0252 & 0.3641 & 0.6721 & 0.0071 \\
5 & 0.4390 & 0.8442 & 0.0058 & 0.0095 & -0.1865 & 0.0068 \\
6 & 0.1854 & -0.4634 & 0.0052 & 0.4359 & 0.0581 & -0.0183 \\
7 & -0.5860 & -0.6109 & -0.0098 & -0.5614 & -0.7011 & -0.0180 \\
8 & 0.2784 & -0.6257 & -0.0095 & 0.2329 & -0.7143 & -0.0054 \\
9 & -0.4336 & -0.6525 & 0.0063 & -0.5824 & -0.7080 & -0.0060 \\
10 & -0.5619 & 0.1653 & -0.0229 & -0.8281 & 0.2557 & 0.0063 \\
11 & 0.3664 & 0.1633 & -0.0014 & 0.4547 & 0.2564 & -0.0030 \\
12 & 0.3545 & 0.1745 & -0.0028 & 0.4460 & 0.2665 & -0.0011 \\
13 & 0.1428 & 0.2269 & -0.0015 & 0.2385 & 0.2899 & -0.0200 \\
14 & 0.4140 & 0.4133 & & 0.4924 & 0.4881 & \\
15 & 0.2110 & 0.3774 & & 0.2710 & 0.4742 & \\
Total & 0.0360 & -0.0059 & -0.0301 & 0.0701 & -0.0060 & -0.0641 \\
\hline
\end{tabular}

${ }^{\mathrm{a}}$ See Fig. 2a.

${ }^{\mathrm{b}}$ Total charge on the surrounding water molecule. 